\title{
A Multi-Tenant Platform for SMS INTEGRATED SERVICES
}

\author{
Ka Ching Chan ${ }^{1}$ and David Tien ${ }^{2}$ \\ ${ }^{1}$ Department of Computer Science and Computer Engineering, La Trobe University, \\ Bendigo, Victoria, Australia \\ ${ }^{2}$ School of Computing and Mathematics, Charles Sturt University, \\ Bathurst, New South Wales, Australia
}

\begin{abstract}
This paper presents the design and development of a Linux based infrastructure and platform for a wholesale service provider to offer SMS integrated services to retail providers for resale. The multi-tenant platform, capable of providing multiple types of SMS integrated services, was designed and built mainly with off-the-shelf components and open source software. The platform is highly reliable and flexible, enabling fast provision of customisable services by integrating SMS, voice, VoIP, and web services in innovative ways. This paper describes the design of the platform and presents four major application areas.
\end{abstract}

\section{KEYWORDS}

Short Message Service, SMS, GSM, Mobile Service

\section{INTRODUCTION}

Short Message Service (SMS) has proven to be popular worldwide in sending and receiving alphanumeric messages on mobile phones, and is supported by all mobile phones from the early GSM mobile handsets to the latest smart phones [1]-[3]. A single SMS message has the length limitation of 160 characters and is delivered within a short time in general. SMS messages can also be delivered internationally. In addition to the common messaging and broadcasting applications, there is a vast amount of research literature published on specialised SMS applications such as diabetes management [4], [5], asthma control [6], [7], weight control [8], mobile payment [9], [10] and other health care applications [11], [12], to name a few. There is a high demand of similar types of low volume, but highly customisable integrated communication services that require some forms of integration of SMS messaging with voice, VoIP, and web applications.

Building on our previous extensive experience in developing custom SMS integrated solutions in the service provider industry, we aimed to design and build a generic platform that meets the demand of this niche market. The presented platform is a wholesale multi-tenant platform that enables fast provision of flexible services to multiple providers for resell to their customer bases. The types of services range from simple outbound SMS broadcasting to any innovative SMS integrated applications. The infrastructure and platform have been built mainly with off-the-shelf components and open source software. Application programming interfaces (HTTP APIs) have been developed to enable internal system communications and external interconnections with customers' systems. A centralized database has been built to support all the system configuration, multi-tenant management, real-time service operations, and billing functions. 


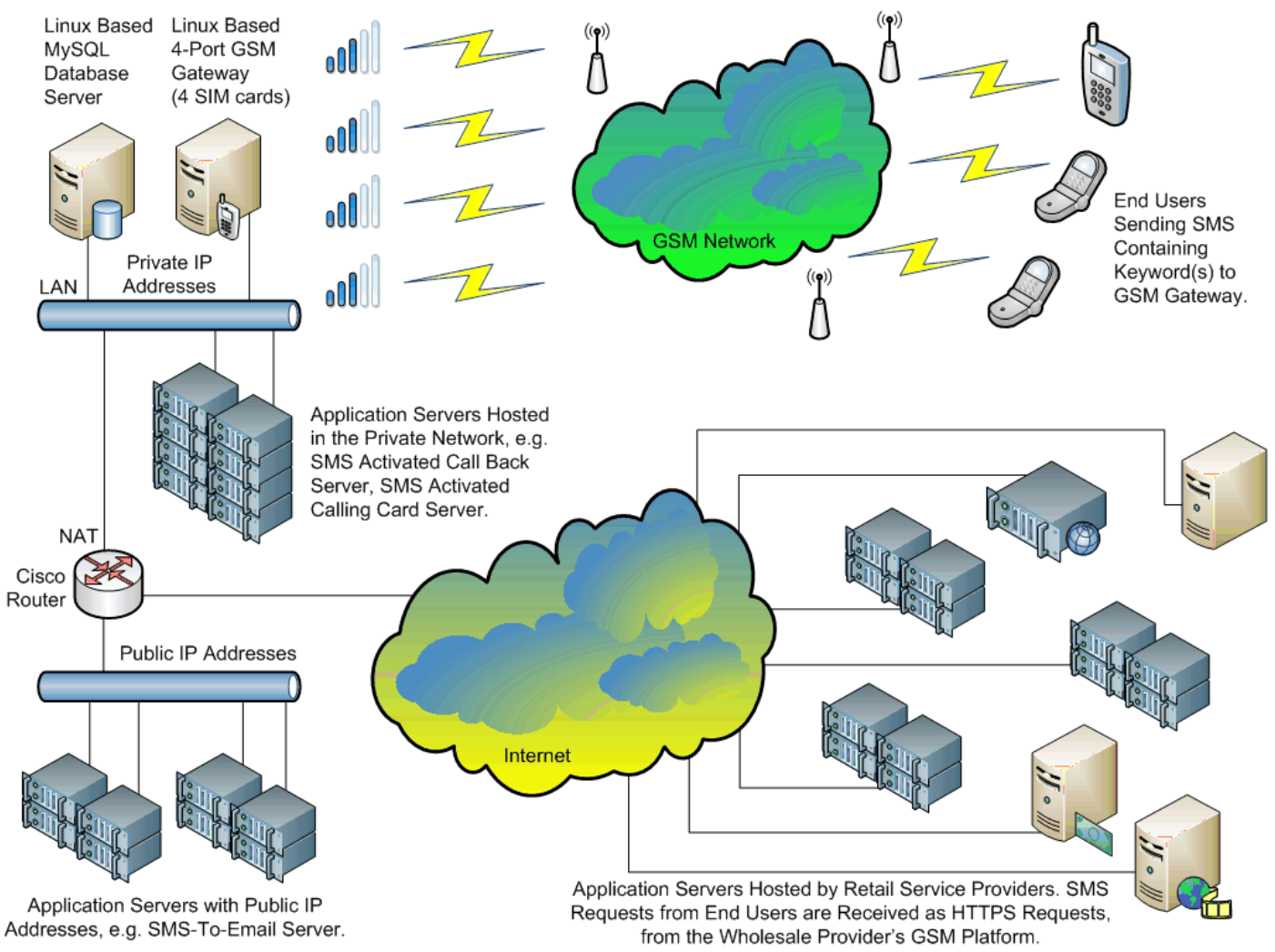

Figure 1. Infrastructure of the SMS integrated multi-tenant platform

\section{SYSTEM ARCHITECTURE}

In this section, we present the overall system infrastructure and development platform on which the wholesale SMS service platform was designed and developed. The infrastructure, involving the integration of various types of servers to offer multiple functionalities and capabilities, enables innovative applications to be built on easily and quickly.

The main systems include a Linux based GSM gateway, a MySQL database server, an Apache web server, and a number of applications servers. The network diagram is given in Figure 1. The GSM gateway, MySQL database server, and application servers are configured with private IP addresses unless public IP addresses are required for communicating with retail providers via the Internet. In the following sections, we will describe the hardware used in our development in more details. For simplicity, equipment used for redundancy and high availability is not included in the diagram.

\subsection{GSM/SMS Gateway}

Direct interconnection with Mobile Network Operators (MNO) is a very high cost approach due to the high charges imposed by the MNOs. Most MNOs will charge a very high setup fee, and recurring monthly fee. This high cost makes it financially unviable for most smaller or new players to enter into any SMS related business. Our approach aims to provide an alternative path for smaller players to enter into the markets, without the need to interconnect with MNOs. 
A GSM gateway provides mobile voice and SMS connectivity to the mobile carrier's GSM network. To build a highly reliable system at the lowest possible cost, we purchased high quality off-the-shelf components and assemble the system ourselves. A special purpose add-on card, the OpenVox G400P GSM modem card, which supports up to four SIM cards, was installed in a dedicated server to build the GSM gateway. This gives us a capacity of up to four simultaneous inbound and/or outbound messages. The G400P is a 32-bit PCI 2.2 compliant card that can be inserted in any PCI slot. Designed for the open source VoIP application Asterisk, G400P enables developers to build powerful and innovative communication applications by integrating the GSM network with the data, VoIP, and PSTN network, on the Asterisk platform.

The device driver for the G400P GSM card, chan_extra, is an open source project supported by OpenVox. The name chan_extra stands for channel driver - the Extended Telephony Technology for Asterisk. It is a software component providing similar functions like chan_sip, chan_zap, and chan_dahdi. As an independent channel driver, chan_extra makes use of the zaptel/DAHDI interface, and adds only necessary components (such as opvxg4xx) without modifying the original zaptel/DAHDI codes. This approach makes it easier to maintain as the need to patch Asterisk every time a new version released is eliminated. The chan_extra and opvxg4xx provides voice and SMS support through the G400P card.

\subsection{Voice/VoIP Telephony Gateways}

The Voice/VoIP telephony gateways are used to interconnect to upstream voice providers or telephone operators for voice termination. They are critical components in the infrastructure in supporting services that require voice communication, such as SMS-to-call or SMS-to-broadcast. Telephony gateways are physical devices that send calls to upstream providers either via PSTN phone lines, or via VoIP through Internet or private data networks. An upstream provider can be a traditional incumbent PSTN telephone operator or VoIP provider. Both Cisco and Asterisk gateways support PSTN and VoIP, and therefore, enable us to send calls to both PSTN and VoIP providers.

There are two types of voice gateways, the Cisco 5350/5400 series gateways and custom built Asterisk gateways, in our backend infrastructure. The Cisco 5350/5400 gateway offers extremely high reliability whilst Asterisk offers high reliability at a significantly lower cost. The Asterisk gateway was built using a Digium PRI card inserted into a PCI slot of a PC server running the open source Asterisk VoIP application. Using a mix of Cisco and Asterisk gateways allows us to achieve high reliability and redundancy at a reduced cost. Both Cisco 5350 and Asterisk gateway supports direct PSTN connection using ISDN PRI, and VoIP.

In the telecommunications industry SIP and $\mathrm{H} 323$ are the main VoIP protocols used for interconnection. Both the Cisco 5350 telephony gateways and the Asterisk VoIP servers support SIP and H323; therefore enabling us to connect to VoIP providers worldwide. In general, the voice quality of VoIP is more prone to fluctuation, especially when sent via the open Internet. However, the capability to send VoIP calls gives us a large choice of potential providers, offering us voice termination at various quality levels and price points. For residential or business grade connections, usernames and passwords are usually required to register end points (i.e. IP phone, or ATA, or softphone) to the providers' SIP servers. For wholesale interconnections, SIP trunks are frequently used. They are direct connections authenticated by IP address. SIP trunks can be setup as private or public connections. For private connections, bandwidth is unlikely an issue and codecs such as G711 alaw or G711 ulaw could be used for the best possible voice quality. G711 alaw and G711 ulaw are uncompressed codecs. For public Internet connections, most providers support G729 and G723 codecs. G729 and G723 are compressed codecs and require only 8K and $6.3 \mathrm{~K}$ bandwidth respectively. 
Due to the interoperability of the Cisco and Asterisk gateways, calls can be made and bridged between the same as well as different protocols. It is possible to make an end-to-end call made up of multiple call legs of PSTN and VoIP (H323 and SIP). In addition, the call legs may use the same or different voice codecs. The Cisco and Asterisk gateways are able to perform codec translations in real time. Selection of the right codecs appropriate for the network environment is important in achieving high voice quality. It is sometimes preferable to use the same codec endto-end if possible, to minimize loss due to compression and decompression as a call traverses multiple voice switches and gateways.

\subsection{Database Servers, Web Servers, and Email Servers}

The platform consists of a number of physical and virtual servers, including a number of VMware ESXi virtualization servers hosting email, web, and application servers as virtual machines, and a number of physical servers. All servers were custom design and built, and most of them ran on the CentOS Linux operating system. In general, virtual servers are preferred except in cases where special hardware is required or there is performance issue. For reliability, redundant network attached servers (NAS), connected to the VMware servers via iSCSI, were built using the open source software Openfiler. All virtual machines are stored and run directly from the Openfiler servers.

The main purpose of the web server is to allow retail providers to connect to our wholesale platform via HTTP API requests. Our platform is flexible and different groups of APIs were developed for different types of service offerings. Service web portals, linked to backend databases, have also been developed for retail providers to setup and manage their own services.

MySQL Database servers were built on dedicated servers rather than as virtual machines for performance reasons. The database servers are the key systems to which other servers link up for information and decision making. The database servers hold information in relation to service configurations, call routing information, service related information such as broadcast lists, user account details, logs, billing details, and call details records, etc. The integrated development environment MySQL Workbench was heavily used in the design, development and maintenance of the databases.

We have custom made a billing system, based on the open source billing software A2Billing and MySQL, to bill retail providers. This billing system enables us to bill once off, recurrent, and usage charges, for different service types. For voice and SMS related services, A2Billing provides great flexibility and a powerful rating engine capable of least cost routing, multi-country support, multi-currency support, inbound and outbound billing, per call/SMS and per time block billing, to name a few.

Email servers are also integral parts in the infrastructure. As email is now one of the standards for the corporate world and personal communications, integrating emails in the wholesale platform opens up many opportunities for innovative applications.

\subsection{Networking Equipment}

The platform was built on a commercial Internet service provider's existing network infrastructure, which was built using Cisco routers and switches. This includes a number of Cisco 7206 routers multi-homed to a number of upstream IP-transit providers with BGP, and a number of Cisco Catalyst series switches for LAN switching and interconnections with retail providers using VLANs. Redundant equipment was setup in two separate data centers in Sydney, linked up with a dark fiber, for high availability. Retail providers who have a presence in one of our data centres may interconnect with us directly using low cost Ethernet, Fast Ethernet, or Gigabit 
Ethernet. Private VLANs and private IP addresses are usually used for these types of connections. Retail providers who are not collocated in the same data centre can still connect to our platform via Internet, with data encryption and proper firewall setup on both sides. Both private and public connections use the same HTTPS API for communications.

\section{SMS INTEGRATED SERVICES}

In this section, we are providing four main types of SMS integrated services that we have supplied on the wholesale platform in the past. These examples demonstrate that the platform is highly flexible enabling service providers to custom design and build their innovative SMS integrated applications for their customer bases.

\subsection{Monitoring and Data Collection Applications}

One of the most popular SMS application areas is monitoring and data collection. In e-health research, SMS has been widely used in diabetic, asthma, and weight, and appointment management systems [4]-[8]. Figure 2 below shows the infrastructure and integral systems in building this type of applications.

As an example, an online diabetes monitoring and management system where patients' blood sugar levels are received as SMS messages or via the website, and stored in a common backend MySQL database. In general, the platform supports any websites that may require data collection via SMS messages. Examples include SMS based voting systems, sports ladder systems, betting systems, and lucky draw systems.

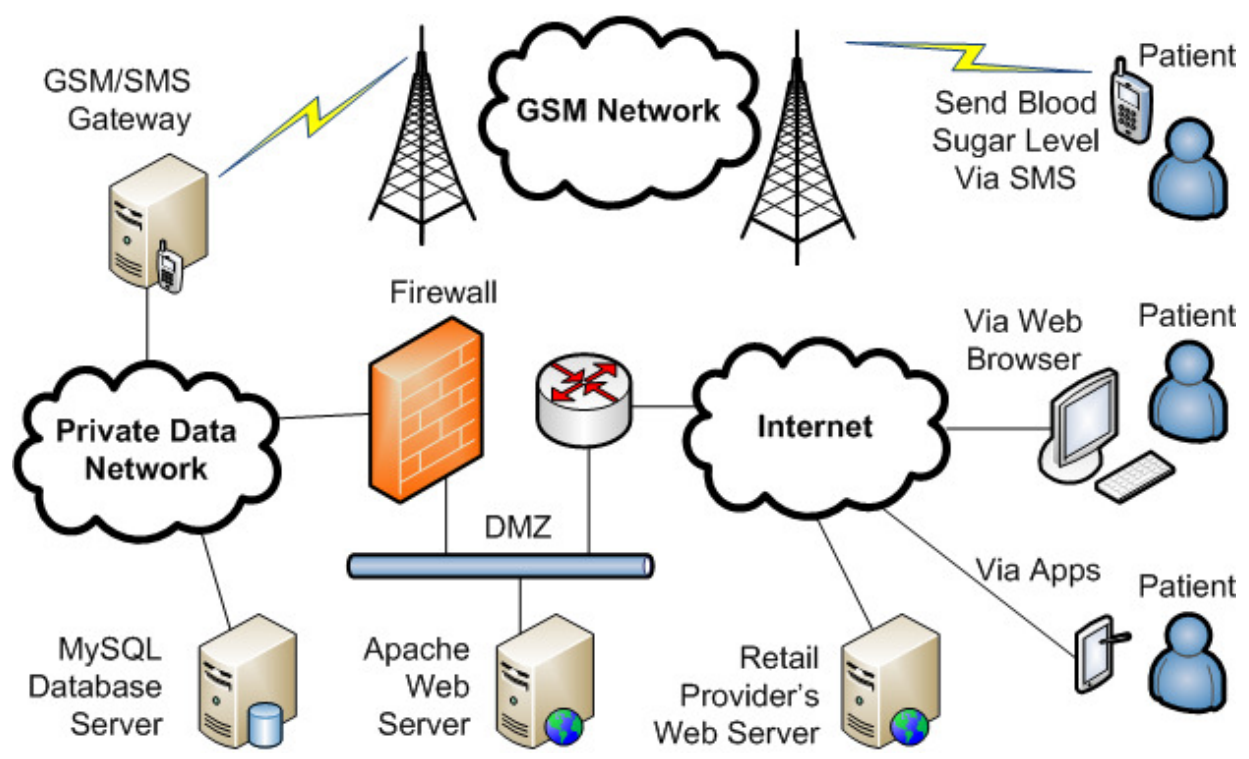

Figure 2. Configuration for monitoring and data collection applications

\subsection{Voice and VoIP Applications}

Figure 3 below shows the infrastructure and systems that are required to offer integrated voice/VoIP and SMS services. In this setup, the Cisco and Asterisk voice/VoIP gateways are essential for making voice calls to the PSTN network. A typical application is SMS-to-call in which a customer sends a SMS message to request a call back. As an example, when our system 
receives a SMS message, e.g. "bankX homeloan", the voice/VoIP gateway will first make a call to bankX's call center responsible for home loan enquiries. Once an operator answers the phone, the gateway immediately makes a call to the customer's mobile phone to bridge the two call legs to establish a telephone conversation.

The integration of voice capability to other services was through the use of Asterisk call files. When a HTTP API request to make a scheduled call is received, the Apache web server will immediately generate and transfer a call file to the Asterisk server. Asterisk call files are structured text files which, when moved to the appropriate directory, are able to automatically place calls. The Asterisk call file can therefore be considered as the key element in bridging the data world to the voice world.

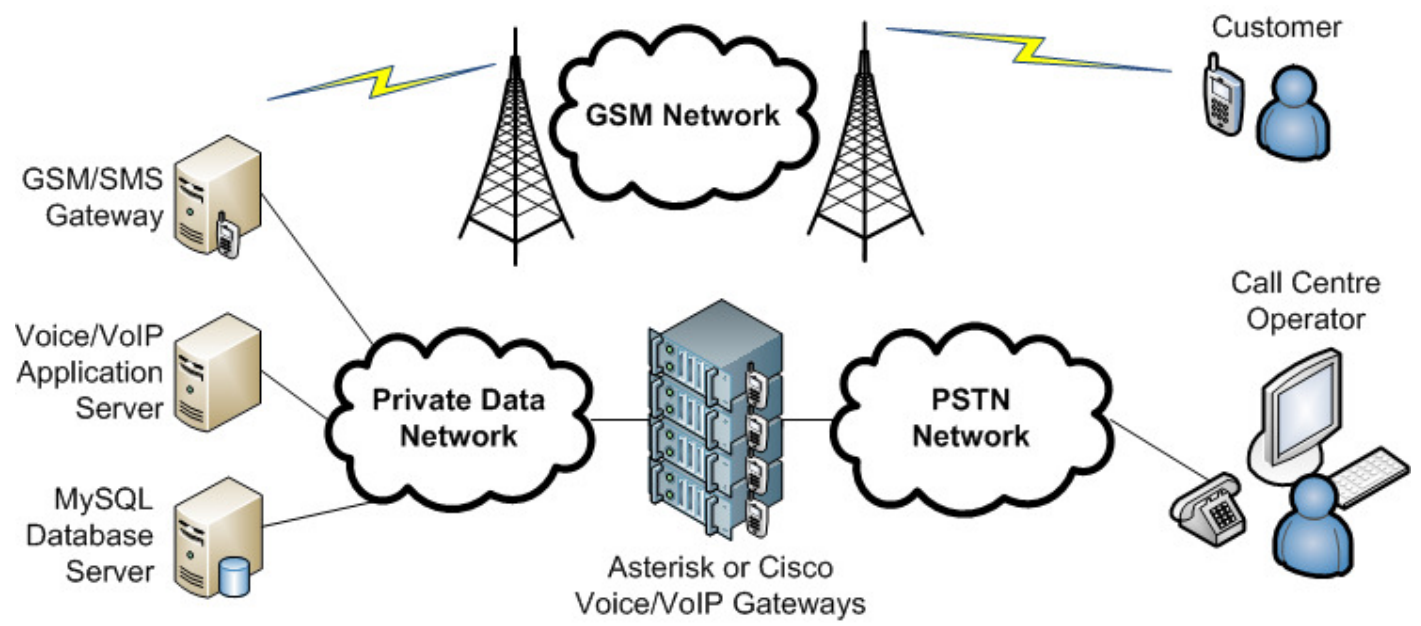

Figure 3. Configuration for voice and VoIP applications

The following services can also be provided via this setup:

- SMS-to-follow-me, e.g. a user sends a SMS message for an enquiry, our platform will ring multiple telephone numbers one by one (call hunting), or simultaneously (ring all) until an operator answers the call. The phone numbers are preset in the database via web portals. This service can also be used by frequent international travelers or mobile workforce whose phone numbers change frequently.

- Click-to-call or web callback service [13], e.g. a user clicks on a button on a website to enter a phone number to receive a call back from the website operator. This service is similar to SMS-to-call except the calls are activated through a website instead of SMS. Our wholesale platform provides a HTTP API to support both the SMS-to-call and clickto-call services.

- SMS and IVR payment services. Payment instructions are received via SMS or IVR with PIN authentication.

- SMS-to-speech, where a SMS text message is converted to speech or voice prompts for broadcasting or replay as IVRs using the voice/VoIP gateways. Our system supports male and female voices, and American, British, and Indian accents.

- Other voice/VoIP enabled services such as call recording, IVR, and follow-me.

\subsection{Transaction Verification Applications}

Figure 4 shows the configuration of the wholesale platform for e-commerce transaction verification applications. For this type of applications, the website owner generates a random 
string or verification code, extracts the mobile phone number of the user requesting the online transaction from the database, and passes them to our platform via a HTTP API. As the wholesale provider, we do not have any private information in relation to the end user. This information is usually collected via the website under the control of the retail provider. All our platform does is to send the verification code to the mobile phone number using the GSM gateway, and to keep records for billing purpose. Once the SMS verification code is received, the end user can enter that to complete the online transaction. Typical applications include online registrations and payment transfer. In general, this configuration can be used to support any kind of online transactions and e-commerce that require SMS verifications. Similarly, it can be used for any online process to withhold and send certain key information via SMS for better security.

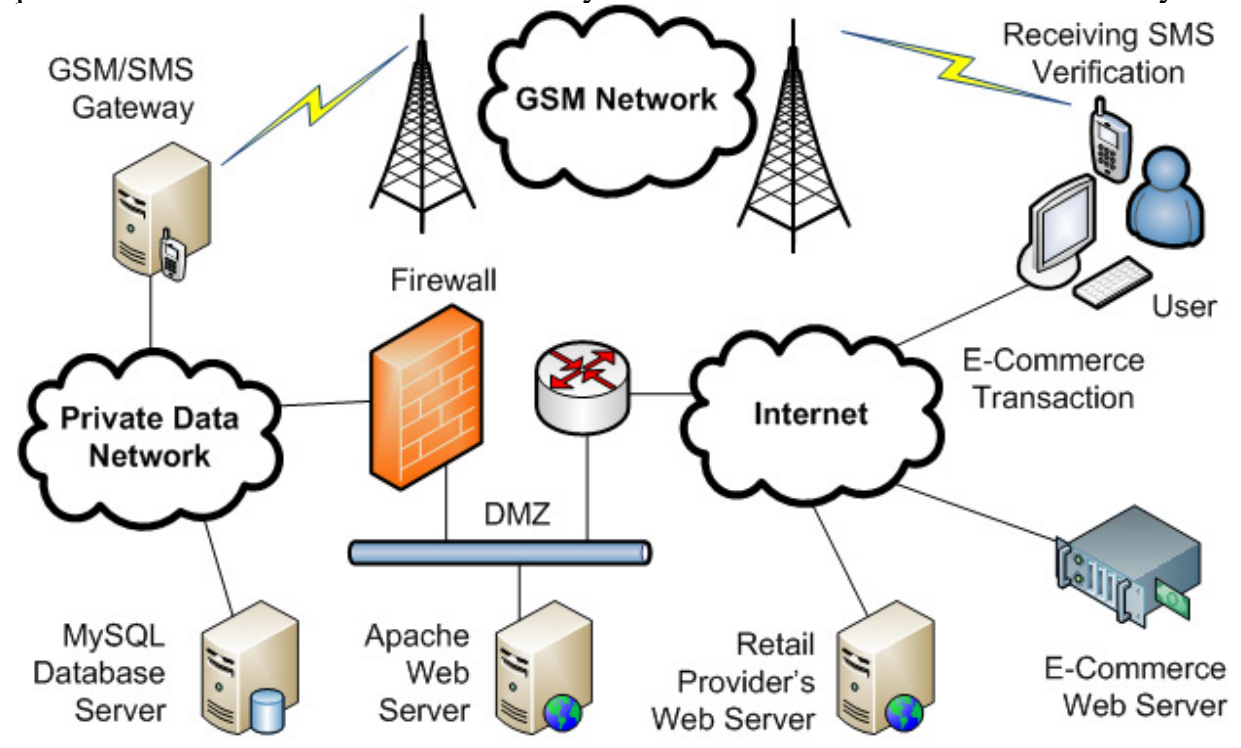

Figure 4. Configuration for e-commerce transaction verification applications

\subsection{International SMS and Broadcast Applications}

Figure 5 below shows the infrastructure needed to offer services that sending outbound domestic and international SMS messages is required. Due to the limited capacity of the GSM gateway, our platform interconnects with a number of upstream SMS carriers to terminate outbound SMS traffic. Interconnection with upstream carriers can be established easily via HTTP API requests through Internet. These carriers can provide international SMS termination on per message sent basis. Depending on the destinations, the rates vary and can be expensive.

A typical HTTP request will include a number of parameters, such as username, password, sender mobile number, receiver mobile number, and the message content. The HTTP requests may be unencrypted or encrypted depending on the type of connections. It is possible to schedule a SMS by adding a date/time parameter. After a text message is sent, the upstream carrier will reply with a delivery receipt as an email or HTTP response with message ID, status, and error message if any, to one of our Apache web servers. Following is a typical HTTP request:

http://www.smsproviderx.com/api.php? mode $=$ sendsms\&username $=x x x \&$ password $=x y z \&$ from $=6$

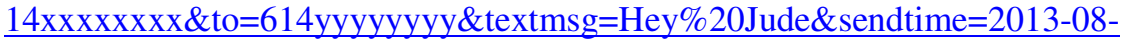
$18 \% 2018 \% 3 \mathrm{~A} 18 \% 3 \mathrm{~A} 18$ 


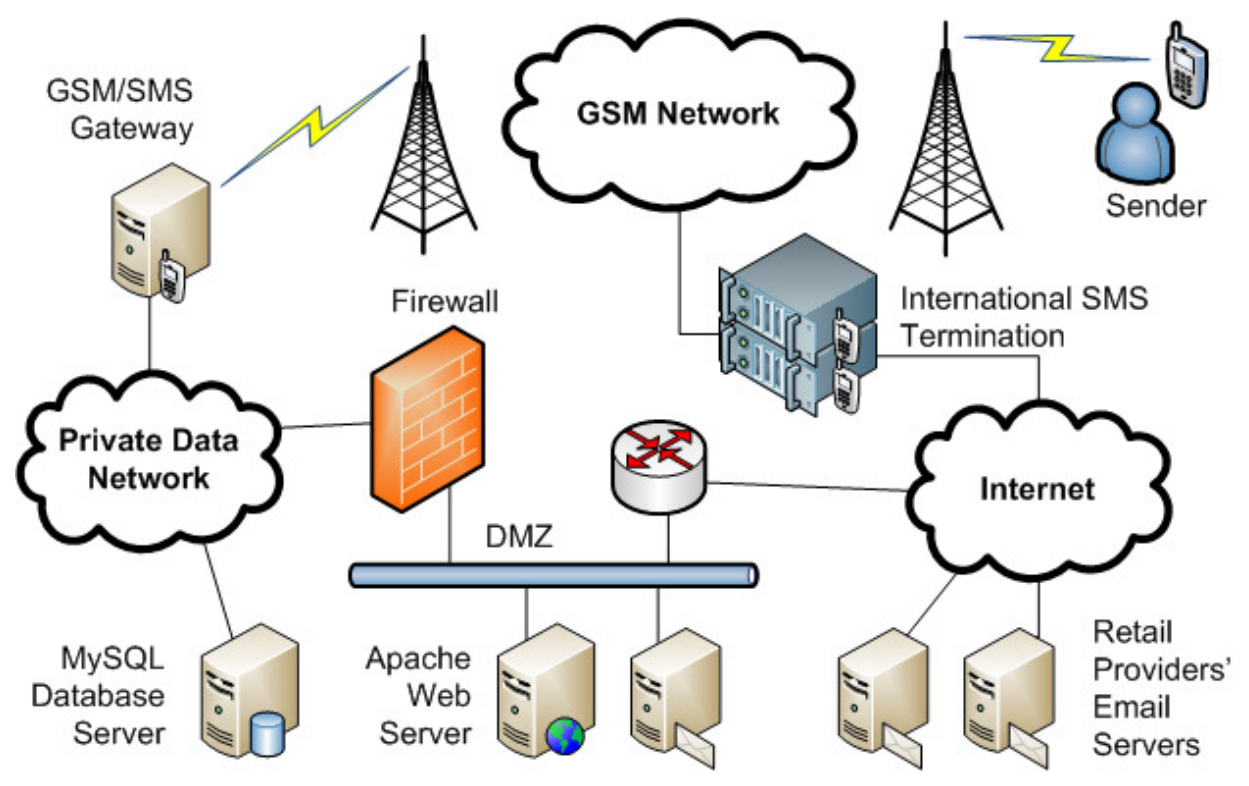

Figure 5. Configuration for broadcast applications

As a wholesale provider, the role is to receive and relay the SMS messages to upstream providers for termination. A few applications making use of this configuration are given below:

- Information delivery via SMS, e.g. a car sales website sending seller and car details via SMS when a potential buyer clicks on a hyperlink.

- Single or bulk SMS-to-SMS, e.g. a soccer coach sending a SMS with the content "grade1 match cancelled" to notify all first grade players that the match is cancelled.

- Single or bulk web-to-SMS, e.g. emergency services using a web portal to enter and broadcast messages to residents of certain areas. Upstream SMS termination providers are used for high volume message broadcasts.

- Single or bulk email-to-SMS, e.g. a user sends an email to phone_noX@email2sms.isp.com and the owner of phone_noX receives the email as a SMS message. For broadcasting an email is sent to groupX@email2sms.isp.com for a pre-defined list of phone numbers associated with groupX.

- Single or bulk SMS-to-email. This type of services costs much less due to email rather than SMS delivery.

- SMS marketing with QR codes. When a potential customer scans a QR code, his/her phone number and the pre-written text message will be sent back to the marketer.

- SMS reminder and notification systems.

\section{Conclusions}

In this paper, we have presented a wholesale platform for service providers to develop and offer SMS integrated services. A wide range of innovative services have been developed and offered by a number of service providers. The platform has been proven to be flexible, scalable and reliability. 
International Journal of Computer Networks \& Communications (IJCNC) Vol.5, No.6, November 2013

\section{ACKNOWLEDGEMENTS}

The authors would like to thank the Australian Government, Department of Innovation Industry, Science and Research, AusIndustry for its financial support through the Innovation Australia R\&D Tax Concession scheme.

\section{REFERENCES}

[1] J. Brown, B. Shipman, and R. Vetter, "SMS: The short message service," Computer, IEEE, vol. 40, no. 12, pp. 106-110, December, 2007.

[2] G. Peersman, P. Griffiths, H. Spear, S. Cvetkovic, and C. Smythe, "A tutorial overview of the short message service within GSM," Computing \& Control Engineering Journal, vol. 11, no. 2, April 2000, pp. 79-89.

[3] M. R. Power and D. Power, "Everyone here speaks TXT: Deaf people using SMS in Australia and the rest of the world," Journal of Deaf Studies and Deaf Education, vol. 9, no. 3, 2004, pp. 333343.

[4] H. S. Kwon, J. H. Cho, H. S. Kim, J. H. Lee, B. R. Song, and J. A. Oh, et al., "Development of web-based diabetic patient management system using short message service (SMS)," Diabetes Research and Clinical Practice, vol. 66S, pp. S133-S137, 2004, doi:10.1016/j.diabres.2003.10.028.

[5] K. C. Chan, L. Wong, and D. B. Chan, "Design of a large scale community based selfmanagement system for diabetes mellitus," in Studies in Health Technology and Informatics, vol. 182, pp. 58-66, November, 2012, doi:10.3233/978-1-61499-152-6-58.

[6] J. Anhøj and C. Møldrup, "Feasibility of collecting diary data from asthma patients through mobile phones and SMS (short message service): response rate analysis and focus group evaluation from a pilot study," Journal of Medical Internet Research, vol. 6, no. 4, December, 2004, doi:10.2196/jmir.6.4.e42.

[7] V. Ostojic, B. Cvoriscec, S. B. Ostojic, D. Reznikoff, A. Stipic-Markovic, and Z. Tudjman, "Improving asthma control through telemedicine: a study of short-message service," Telemedicine Journal and E-Health, vol. 11, no. 1, 2005, pp. 28-35.

[8] N. S. Joo and B. T. Kim, "Mobile phone short message service messaging for behaviour modification in a community-based weight control programme in Korea," Journal of Telemedicine and Telecare, vol. 13, no. 8, 2007, pp. 416-420.

[9] S. Fong and E. Lai, "Mobile mini-payment scheme using SMS-credit," in Computational Science and Its Applications - ICCSA 2005. Lecture Notes in Computer Science (3481), Berlin, Heidelberg: Springer, 2005, pp. 1106-1114.

[10] H. Harb, H. Farahat, and M. Ezz, "SecureSMSPay: Secure SMS Mobile Payment model," in Proc. 2nd Int. Conf. Anti-Counterfeiting, Security and Identification, 2008, pp. 11-17.

[11] B. S. Fjeldsoe, A. L. Marshall, and Y. D. Miller, "Behavior change interventions delivered by mobile telephone short-message service," American journal of preventive medicine, vol. 36, no. 2, 2009, pp. 165-173.

[12] R. T. Lester, P. Ritvo, E. J. Mills, A. Kariri, S. Karanja, and M. H. Chung, el al., "Effects of a mobile phone short message service on antiretroviral treatment adherence in Kenya (WelTel Kenya1): a randomised trial," The Lancet, vol. 376, no. 9755, November 2010, pp. 1838-1845.

[13] K. C. Chan, "A wholesale web callback service," in Proc. $8^{\text {th }}$ Int. Conf. Information Technology and Applications (ICITA 2013), IEEE, Sydney, Australia, 1-4 July, 2013. 


\section{Authors}

Ka Ching Chan received his BASc in Engineering Science and MASc in Mechanical Engineering from the University of Toronto, Canada, and his $\mathrm{PhD}$ in Manufacturing Engineering from the University of New South Wales, Australia. He was previously a Senior Lecturer at the University of New South Wales, and a Visiting Assistant Professor at the Hong Kong University of Science and Technology. Dr. Chan also had extensive industry

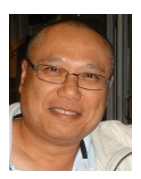
experiences as founders and CTOs in growing technology companies from startups to public companies. Dr. Chan is currently a Lecturer in Information Technology at La Trobe University and a Visiting Fellow in Mechatronics at the University of New South Wales. He is currently serving as the Secretary of the IEEE Computer Society, NSW Chapter.

David Tien received his undergraduate, master's and Ph.D. degrees in Computer Science, Pure Mathematics and Electrical Engineering from Harbin, Chinese Science Academy, the Ohio State University, and the University of Sydney, respectively. Prior to joining the School of Computing and Mathematics, Charles Sturt University in 2000, he had 20 years' experience researching and lecturing at the University of Sydney, Ohio State University and Singapore. Currently, his major research interests are GIS, image and signal processing, telecommunication, education theory, and biomedical engineering. During the past 25 years, David served as the Chairman, Secretary, Treasurer and Chairman of the IEEE Sections in Australia and Singapore, and is currently the Chairman of NSW Computer Chapter. David also served as an elected member of the Charles Sturt University Council. David enjoys working and travelling. Currently, he has one child, one wife, and one dog who rules the house. 